\title{
Volatility DyNAMics of NYMEX NATURAl GAS Futures Prices
}

\author{
Hiroaki Suenaga* \\ Research Fellow \\ School of Economics and Finance \\ Curtin Business School \\ Curtin University of Technology \\ Aaron Smith \\ Assistant Professor \\ Department of Agricultural and Resource Economics \\ University of California, Davis \\ and Member, Giannini Foundation \\ Jeffrey Williams \\ Daniel Barton DeLoach Professor \\ Department of Agricultural and Resource Economics \\ University of California, Davis \\ and Member, Giannini Foundation \\ * Correspondence to: Hiroaki Suenaga, School of Economics and Finance, Curtin University of Technology, GPO Box \\ U1987, Perth, WA 6845, Australia. (e-mail: hiroaki.suenaga@cbs.curtin.edu.au).
}

\begin{abstract}
Despite their importance in pricing futures and other derivative contracts, seasonal variations in mean and variance of energy prices have not been fully captured in previous studies of energy prices. We examine the volatility dynamics of daily natural gas futures traded on the NYMEX via the partially overlapping time-series (POTS) model of Smith (2005, Journal of Applied Econometrics). We illustrate that the volatility of daily price changes of natural gas exhibits strong seasonality, even as the volatility increases as a contract approaches its expiration, a time-to-maturity effect. Our analysis reveals that the persistence of price shocks and, hence, the correlations among concurrently traded contracts, also exhibit substantial seasonal and cross-sectional variation. These volatility patterns we estimate are closely related to the seasonal cycle of US natural gas storage in a way consistent with the theory of storage. We demonstrate that, by ignoring the seasonality in the volatility dynamics of natural gas futures prices, previous studies have suggested sub-optimal hedging strategies.
\end{abstract}




\section{INTRODUCTION}

Natural gas demand in the US peaks around December to March with the demand for residential heating. While this unremarkable fact of winter weather implies higher average prices in that season, the seasonality in the weather itself is mitigated in prices through intertemporal arbitrage, to the level in the off-peak demand period (spring and summer) plus the cost of carry. Specifically, the theory of storage asserts that the equilibrium constellation of spot and futures prices represents where the marginal benefit of current consumption is equal to the expected marginal benefit of storing a commodity for future consumption. This price relationship does not hold between two contracts if inventory is effectively zero at any point between their maturity dates.

The typical seasonality in the constellation of natural gas is illustrated in Figure 1, displays all forty-six futures prices on the NYMEX on April 1, 2003. Those many contracts, although stretching far into the future, are re-aligned in Figure 1 to the conventional April-March year in natural gas. That realignment by month from April reveals the strong increase in price during the fall and early winter, precisely when stocks are peaking. Unlike for many commodities, storage facilities for natural gas can reach collective capacity, which implies price relationships can imply a substantial marginal cost to storage and effective seasonality in that marginal cost.

Strong seasonality in demand and storage also implies highly non-linear volatility dynamics to natural gas prices. Volatility is naturally high for winter contracts because the marginal cost of natural gas production is high and demand, supply, and other market shocks of even a small magnitude can cause in a large price swing. At the same time, high natural gas inventory during winter months reduces volatility of the early winter contracts, for price shocks can be absorbed, albeit partially, by releasing or absorbing inventory. Such flexibility is inevitably lower later in the winter. The seasonal storage pattern also implies that prices of winter contracts start fluctuating as early as the preceding off-peak demand period, during which information arrives about the future availability of stored gas. In contrast, prices of spring and summer contracts should not exhibit substantial movements until the end of the preceding peak demand season because little inventory is carried over from winter to spring in a normal year.

A recent increase in level and volatility of natural gas prices has raised the importance of better understanding of its price dynamics and tools to hedge against price risk. Although 
pricing of futures and other derivative contracts, as such tools, requires proper understanding of the volatility dynamics of energy commodity prices, much of previous research on natural gas and other energy price dynamics has not accounted for complex volatility dynamics resulting from peculiarities of these commodities. Two approaches have been common to previous studies of energy futures prices. The first approach is to construct models of energy price dynamics, in which the spot price is specified as a function of underlying state variables following stipulated stochastic processes. ${ }^{1}$ The suggested model is then used to derive the valuation formula of futures and other derivative contracts, any difference from the observed futures prices being interpreted as representing risk premium. Manoliu and Tompaidis (2002), for example, applied one- and two-factor models to NYMEX natural gas futures prices and reported that these prices exhibit strong seasonal variation while deviation from this seasonal mean reverts to zero.

The second approach has been to examine directly the relationships between the spot and futures prices without stipulating the stochastic processes of the observed price series. Two questions are commonly addressed in these studies: (i) if the futures price is unbiased in forecasting subsequently realized spot price and (ii) if the spot price and the prices of concurrently traded futures contracts exhibit long-run relationships and, if so, how quickly they converge to the equilibrium when they deviate. The first question is usually addressed by examining the statistical significance of the difference between the spot (nearby futures) and futures prices. Several studies have reported that the NYMEX natural gas futures prices are downward biased in forecasting the subsequently realized spot price and interpreted this bias as representing a risk premium (Walls, 1995; Modjahedi and Movassagh, 2005; Movassagh and Modjahedi, 2005). For the second question, Root and Lien (2003) and Lien and Root (1999) have examined the long-run relationships between the spot price and the prices of concurrently traded futures contracts by various cointegration methods and concluded that these prices share a common stochastic trend and respond to deviations from their long-run relationships in such a way that the difference between these prices, or basis, converges to zero. ${ }^{2}$

Yet improper understanding of volatility dynamics resulting from peculiarities of natural gas as a commodity traded in the market has potentially led these studies to erroneous conclusion on

\footnotetext{
${ }^{1}$ For empirical analyses of other energy prices, see, for example, Schwartz (1997) for the NYMEX crude oil and Lucia and Schwartz (2002) for the Nordpool wholesale electricity market.

${ }^{2}$ More precisely, they concluded that the spot price and prices of simultaneously traded futures prices are in one-to-one relationships, or, in other words, zero basis.
} 
the market risk premium or, more generally, the efficiency of the existing futures markets. Williams and Wright (1991) have shown that the equilibrium futures prices derived from a dynamic rational expectation model of a seasonal storable commodity are highly non-linear and non-smooth, which is to say that they cannot be expressed in a reduced-form. Clearly, specified seasonal price cycles can only approximate the true price dynamics. Strong seasonality in storage implies that the difference between the spot price and prices of concurrently traded futures contract, or the basis, representing cost of carry, varies across contract delivery months. Previous studies on spot-futures relationships have hardly incorporated such seasonal variations in basis.

Aside from these modeling issues, previous studies utilized only a subset of price data available form organized exchanges where multiple contracts with different maturity dates are concurrently traded. The test of bias in futures price is usually performed using a monthly data series constructed by stacking the futures prices from some particular day of the month. ${ }^{3}$ Another practice is to construct a daily series by splicing together the nearby futures contracts. Such practices not only discard much information but also distort the temporal dynamics of the observed price series due to switching from one delivery month to another. ${ }^{4}$

In this paper, we illuminate these issues through examining the volatility dynamics of the NYMEX natural gas futures prices using the partially overlapping time series (POTS) model recently suggested by Smith (2005). Unlike conventional models of commodity price dynamics, the POTS model, when applied to the first difference of commodity futures price, does not specify seasonal or any other deterministic variations in commodity futures price and, hence, is much less susceptible to misspecification bias. The POTS model incorporates seasonal and crosssectional variations in the factor loadings and in an idiosyncratic variance. These flexible specifications can capture the time-to-maturity effect, seasonal volatility, and other non-linear volatility dynamics resulting from peculiarities of the natural gas as a commodity.

\footnotetext{
${ }^{3}$ This practice is particularly common to the test of futures price bias, for most energy futures contracts are defined in monthly blocks.

${ }^{4}$ Testing for bias in futures prices with a small sample size is problematic for the OLS estimates of coefficients in the regression of spot price on futures price are biased and consistent due to the correlation between the current forecast error (spot minus futures price) and the prices of subsequently traded futures contracts (Williams and Wright, 1991). Besides, natural gas futures price, both spot and futures, follow nearunit root process, for which widely used unit-root tests, such as ADF, fail to reject the non-stationarity, especially in a small sample. Suenaga and Williams (2005) have shown that the bias in the OLS estimates is of considerable magnitude and even greater for the dynamic OLS for the sample size available from the NYMEX natural gas market.
} 
To illustrate the practical importance of these issues, we apply our analysis of the volatility dynamics of natural gas futures prices to the standard "optimal hedging" strategy. We illustrate that seasonal and cross-sectional variations in the degree of persistence of the price shocks together with the seasonality in the arrival of information, imply that the December and summer (June through August) contracts are more effective than other contracts in minimizing the variance of portfolio return. Previous studies of energy price dynamics, not incorporating seasonal and cross-sectional variations in volatility of natural gas prices, have suggested the use of the adjacent delivery contract.

\section{Partially Overlapping Time-Series Model}

The partially overlapping time-series (POTS) model, recently introduced by Smith (2005), is a latent factor model of daily futures price changes. In a general $k$-factor setting, the model takes the following form,

$$
\Delta \mathbf{F}_{t}=\theta_{t} \varepsilon_{t}+\lambda_{t} \mathbf{u}_{t}
$$

where $\Delta \mathbf{F}_{t}$ is an $n_{t}$ by one vector of daily futures price changes with $n_{t}$ representing the number of contracts traded on day $t$. It comprises $\Delta F_{d, t}=F_{d, t}-F_{d+1, t-1}$ where the two subscripts represent the number of days before maturity and trading date, respectively. $\varepsilon_{t}$ is $k$ by one vector of latent factors with $\mathrm{E}\left[\boldsymbol{\varepsilon}_{t} \boldsymbol{\varepsilon}_{t}^{\prime}\right]=\mathbf{I}$ and $\theta_{t}$ is $n_{t}$ by $k$ matrix of factor loadings. $\mathbf{u}_{t}$ is $n_{t}$ by one vector of idiosyncratic error with its component, $u_{d, t} \sim \mathrm{N}(0,1)$ and $\lambda_{t}$ is $n_{t}$ by $n_{t}$ diagonal matrix determining the variance of idiosyncratic error.

The components of the matrices, $\theta_{t}$ and $\lambda_{t}$, are specified as a function of maturity date, $m=t+$ $d$, and time to maturity of contract. That is,

$$
\begin{aligned}
& \theta_{d, i, t}=\theta_{i}(d, m) \\
& \lambda_{d, t}=\lambda(d, m)
\end{aligned}
$$

For identification, it is assumed that $\mathrm{E}\left[\varepsilon_{i, t^{2}}\right]=1$ for all $i=1, \ldots, k, \mathrm{E}\left[\varepsilon_{i, t}, \varepsilon_{i, t}\right]=0$ for all $i \neq j, \mathrm{E}\left[\varepsilon_{i, t}\right.$ $\left.u_{d, t}\right]=0$ for all $i, d, t$, and $\mathrm{E}\left[\Delta \mathbf{F}_{t} \mid \mathfrak{I}^{t-1}\right]=0$ where $\mathfrak{I}^{t-1}$ denotes the information set available at $t-1$. 
These assumptions assert that series of daily futures price changes follow the Martingale difference sequence, which implicitly assumes a zero risk premium. ${ }^{5}$

Following Smith (2005), we assume that the conditional variance of $\varepsilon t$ follows $\operatorname{GARCH}(1,1)$ process in BEKK specification (Engle and Kroner, 1985),

$$
\mathrm{E}\left[\boldsymbol{\varepsilon} t \boldsymbol{\varepsilon}^{\prime} \mid \mathfrak{J}^{t-1}\right]=\mathbf{H}_{t}=\omega+\boldsymbol{\beta} \mathbf{H}_{t-1} \boldsymbol{\beta}^{\prime}+\alpha E\left[\boldsymbol{\varepsilon}_{t-1} \boldsymbol{\varepsilon}_{t-1}^{\prime} \mid \mathfrak{J}^{t-1}\right] \boldsymbol{\alpha}^{\prime}
$$

Since the unconditional variance of $\varepsilon, t$ is unity, we have $\omega=I-\alpha^{2}-\beta^{2}$. The last term in (3) is,

$$
\mathrm{E}\left[\boldsymbol{\varepsilon}_{t-1^{2}} \mid \mathfrak{J}^{t-1}\right]=\boldsymbol{\varepsilon}_{t-1}\left|t-1{ }^{2}+\mathbf{P}_{t-1}\right| t-1
$$

where $\boldsymbol{\varepsilon}_{t-1} \mid t-1=\mathrm{E}\left[\boldsymbol{\varepsilon}_{t-1} \mid \mathfrak{I}^{t-1}\right]$ and $\mathbf{P}_{t-1 \mid t-1}=\mathrm{E}\left[\left(\boldsymbol{\varepsilon}_{t-1}-\boldsymbol{\varepsilon}_{t-1} \mid t-1\right)^{2} \mid \mathfrak{J}^{t-1}\right]$, which are obtained through the Kalman Filter (Hamilton, 1994).

The POTS model as defined in (1) through (3) is econometrically very similar to factor models of commodity price dynamics, such as Schwartz (1997) and Manoliu and Tompaidis (2002) as applied to natural gas. A major difference is that the POTS model specifies the dynamics of daily price changes whereas commodity price models specify the dynamics of daily prices. Modeling daily price changes, the POTS model does not specify seasonal or any other deterministic variations in the underlying spot price and, hence, is free of approximation bias. This bias is often large in commodity price models, especially when a commodity price under investigation exhibits such a strong seasonal pattern as natural gas.

The POTS model also employs very flexible functional forms in specifying factor loadings and variance of idiosyncratic error. In contrast, factor loadings are determined by a small number of parameters defining the stochastic process of underlying factors in a majority of commodity price models. Besides, even though recent advancements in models of commodity price dynamics have been attained by increasing the number of factors or specifying more complex stochastic process for each factor, a simple, usually normal, distribution is still assumed for idiosyncratic errors. ${ }^{6}$ Flexible functional forms employed in the POTS model intend to

\footnotetext{
${ }^{5}$ We also estimated the model relaxing this assumption, allowing the unconditional expectation of daily price changes to be non-zero. However, the estimate of the mean daily price change is very small in value while the model's implications for the volatility dynamics of natural gas futures price do not differ from the model assuming zero risk premium.

${ }^{6}$ Suenaga (2005) illustrated that this difference in the level of complexity of the stipulated variance structure of latent factors and idiosyncratic errors has substantial impact on the other model parameters including the risk premium.
} 
capture time to maturity effects and seasonal volatility as well as other non-linear volatility dynamics of natural gas futures prices resulting from peculiarities of the commodity.

\section{DATA AND ESTIMATION RESULTS}

\subsection{Data}

We estimated the model (1) and (2) using daily settlement price data from the NYMEX natural gas futures markets. The NYMEX started trading natural gas futures contracts in April, 1990. Contracts traded in this market are defined in monthly block with each contract providing for the delivery of 10,000 million British Thermal unit (BTu) of natural gas at the Henry Hub located in Louisiana. The market initially traded contracts as far as 1 year before the first calendar day of the delivery month, which was later extended to 6 years. Contracts were traded until seven business days prior to the first calendar day of the delivery months, which was later extended to 3 business days prior to the delivery month.

We analyzed the daily settlement prices from January 2, 1991 to December 31, 2003. These price series are transformed into log price series. Because distant delivery contracts are not always actively traded, we dropped contracts of more than 12 months to maturity. Excluding these observations, we have a sample of 40,618 prices among 175 contracts.

\subsection{Estimation Results}

In applying the model to the NYMEX natural gas futures price data, we specified the factor loadings and the variance of idiosyncratic error in (2) by the following trigonometric functions,

$$
\begin{aligned}
& \theta_{d, m}=\theta^{n}(d)=a_{0}^{m}+a_{1}^{m} d+\sum_{j=1}^{5}\left(a_{2 j}^{m} \sin \left(\frac{2 \pi j d}{d_{\max }}\right)+a_{2 j+1}^{m} \cos \left(\frac{2 \pi j d}{d_{\max }}\right)\right) \\
& \lambda_{d, m}=\lambda^{\mathrm{m}}(d)=b_{0}^{m}+b_{1}^{m} d+\sum_{j=1}^{5}\left(b_{2 j}^{m} \sin \left(\frac{2 \pi j d}{d_{\max }}\right)+b_{2 j+1}^{m} \cos \left(\frac{2 \pi j d}{d_{\max }}\right)\right)
\end{aligned}
$$


where $d_{\max }=365$ for all 12 contracts $(m=1, \ldots, 12) \cdot{ }^{7}$ We estimated the model by the method of Maximum Likelihood with the initial value obtained by the iterative method of Smith (2005). ${ }^{8}$

Table 1 summarizes the coefficient estimates of the GARCH parameters. In the table, the coefficient estimates of $\gamma$ and $\gamma_{2}$ are 0.0911 and 0.8095 . The sum of two coefficients, 0.9006 , is less than unity, indicating that the conditional volatility of the common underlying factor is highly persistent yet is stationary.

Figure 2 plots, over a range of trading dates, the unconditional variance of daily price changes of each of 12 contracts computed as $\theta_{d, m^{2}}+\lambda_{d, m^{2}}$, using the estimated factor loadings, $\theta_{d, m}$, and variance of idiosyncratic error, $\lambda_{d, m}$. The figure indicates at least three interesting patterns in volatility dynamics of daily natural gas price changes. First, for all 12 contracts, volatility generally spikes during a few months before the maturity date, except that, for the February through May contracts, volatility is the highest in the beginning of January. Such a volatility spike, often called time-to-maturity or Samuelson effect, has been often reported in previous studies of commodity futures prices. Second, volatility during the last few months of trading is substantially higher for winter contracts than for late spring or summer contracts. As previously discussed, high price volatility is naturally expected for winter contracts because demand reaches contemporaneous supply capacity in winter but, at the same time, high inventory during winter allows price shocks of certain magnitudes to be absorbed. High volatility depicted in Figure 2 indicates that such price buffering is only limited. Third, aside from these Samuelson effects and seasonal patterns, volatility appears to increase in two periods. The first period is from early November to mid January where volatility increases for all 12 contracts with the magnitude substantially greater for the January to April contracts. The second period is early May to late September during which volatility increases for all contracts maturing before the beginning of the following off-peak season.

These seasonal patterns in price variance are as expected for the price movements of storable commodities. Temporal arbitrage induces a fair amount of natural gas to be carried over from

\footnotetext{
${ }^{7}$ Specified as in $\left(2^{\prime}\right)$, the two functions become more flexible with the number of trigonometric terms included. While this allows the model to fit better to the observed data, it also makes coefficient estimates more sensitive to the extreme observations.

8 The estimation method involves iteration of the following three steps: (1) obtain the predicted values of the latent factor and GARCH conditional variance through Kalman Filter, (2) maximize the expected likelihood with respect to the spline parameters, conditional on the predicted values of latent factor and GARCH
} 
off-peak demand (April through June) to peak demand season (December to March next year) so that the price difference between peak and off-peak demand season is on average equal to the cost of carry. Figure 3 illustrates such storage pattern. In the figure, the US nationwide working gas storage is the lowest in March, after which it gradually increases throughout spring and summer until it reaches annual high in October or November. A rapid decrease of inventory over November through February following year indicates that a large amount of stored gas is withdrawn to meet with a high demand for heating energy.

As observed in Figure 2, even though prices of winter contracts exhibit enormous volatility during winter trading days, prices of more distant contracts do not move much over the same trading period. For these contracts, volatility increases because current demand-supply imbalance determines how much of inventory is carried over from the current peak demand season to subsequent months (as a result of unexpectedly low demand, for example), yet only slightly so because the amount of gas carried over is very small relative to the amount stored over the off-peak season in a normal year. Volatility increase in late spring (May to late September) also implies the arrival of important market information. During this period, inventory continuously changes, as much of excess natural gas production is stored for the use in subsequent peak demand season. Gas stored in this period will not be carried over to the following off-peak demand season unless gas demand in the coming peak demand season is unusually low. Such information will not be revealed until actual demand and/or supply conditions are realized. Hence, price volatility increases, during May to late September trading periods, only for the contracts deliverable to the coming peak demand season and earlier.

Table 2 summarizes the proportion of the variance explained by the common factor. It indicates that the model explains, on average, about $83 \%$ of daily price variation. In general, spring and summer contracts are closely related to the common factor whereas winter contracts are weakly related to the common factor. Figure 3 illustrates, for each of the 12 contracts, how the proportion of total price variance explained by the common factor changes over the trading period. For a majority of contracts, this proportion drops substantially below $100 \%$ in two cases. First, it drops sharply within a few months before maturity, verifying that a sharp increase in the variance of daily price changes during this period is contract-specific. Aside from this drop representing the Samuelson effect, the share explained by the common factor generally increases

conditional variance from the first step, and (3) estimate the GARCH parameters holding the spline parameters at the values from the step 2 . 
or stays at current level for a majority of contracts in winter and spring trading days. This is the evidence that the information arriving during these periods represents market conditions of both current and subsequent periods. Natural gas storage is the primary example of such information for it determines the availability of natural gas for both current and subsequent months.

Second, for the October to December and January to March contracts, the share explained by the common factor drops substantially around the middle to the end of August and keeps decreasing afterwards. That is, much of the information arriving during this period has contractspecific effect on these contracts. The observation is somewhat puzzling, it may be attributable to the marginal cost of storage that increases with the inventory level. To inject gas into underground storage facility requires gas to be high-pressured and this pressure increases with the amount of gas already in storage. Thus, although high inventory accumulated by the beginning of summer allows current market shocks to be absorbed through adjusting inventory, such buffering is costly, which makes the impact of the underlying market shocks on the futures prices of subsequent delivery less even.

In short, the estimated POTS model illuminates complex volatility dynamics of natural gas futures prices. It revealed that the volatility of natural gas futures price exhibits both Samuelson effect and strong seasonality with volatility higher for winter contracts than for other contracts. The volatility also increases in early November through mid January for the January to April contracts and in early May to September for all contracts maturing by the end of the following peak-demand season. The correlation between the daily price changes of concurrently traded contracts tends to be high during these two periods. Previous studies on natural gas futures prices have not depicted such complex dynamics of natural gas price volatility for their econometric models presumed much simpler volatility specification. Although it is not immediately obvious how such misspecification affects those studies' conclusions, our results suggest that previous studies of natural gas price dynamics and spot-futures price relationships need to be reexamined.

\section{OPTIMAL HEDGING STRATEGY}

In this section, we extend our analysis of the volatility dynamics of natural gas futures prices to implications for a hedging strategy. We consider a simple hedging strategy, in which a hedger has a spot position, $Q$, at time $t$ and, simultaneously sells $X$ futures contact for delivery at $\tau>t$. 
At $t+k<\tau$, the hedger clears its position by selling $Q$ units in the spot market and buying $X$ futures contracts for delivery at $\tau$.

The hedger's wealth at $t+k$, ignoring the interest rate, is,

$$
W_{t+k}=\left(S_{t+k}-S_{t}\right) Q-\left(F_{t+k, \tau}-F_{t, \tau}\right) X=\left(\Delta S_{t+k}-\eta \Delta F_{t+k, \tau}\right) Q
$$

where $S_{i}$ is the spot price at $i, F_{i, \tau}$ is the period $i$ price of the futures contract for delivery at $\tau$, and $\eta=X / Q$ is the hedge ratio. The variance of $W_{t+k}$ is

$$
\mathrm{V}\left[W_{t+k}\right]=\left(\mathrm{V}\left[\Delta S_{t+k}\right]+\eta^{2} \mathrm{~V}\left[\Delta F_{t+k, \tau}\right]-2 \eta \operatorname{cov}\left[\Delta S_{t+k}, \Delta F_{t+k, \tau}\right]\right) Q^{2}
$$

which is minimized by,

$$
\eta_{\mathrm{t}, \tau}^{*}=\operatorname{cov}\left[\Delta S_{t+k}, \Delta F_{t+k, \tau}\right] / \mathrm{V}\left[\Delta F_{t+k, \tau}\right]
$$

Substituting (6) into (5) yields the minimized variance,

$$
\mathrm{V}\left[W_{t+k} \mid \eta_{t, \tau}^{*}\right]=\mathrm{V}\left[\Delta S_{t+k}\right]\left(1-\rho_{t+k, \tau}^{2}\right) Q^{2}
$$

where $\rho_{t+k, \tau}$ is the correlation between spot and futures contract for delivery $\tau$ in their price changes over period $t$ to $t+k$.

Alternatively, for a hedger minimizing the variance of the portfolio return, $r=r_{S}-\eta^{r} r_{F}$, the optimal hedge ratio and the associated minimum variance are,

$$
\begin{aligned}
& \eta_{t, \tau}^{r^{*}}=\operatorname{cov}\left[\Delta \ln S_{t+k}, \Delta \ln F_{t+k, \tau}\right] / \mathrm{V}\left[\Delta \ln F_{t+k, \tau}\right] \\
& \mathrm{V}\left[r_{t+k} \mid \eta_{t, \tau}^{r^{*}}\right]=\mathrm{V}\left[\Delta \ln S_{t+k}\right]\left(1-\rho_{t+k, \tau}^{r}{ }^{2}\right) Q^{2}
\end{aligned}
$$

where $\rho_{t+k, \tau}^{r}$ represents the correlation between the log spot price and log price of futures contract for delivery at $\tau$ over the period $t$ to $t+k$.

Three remarks should be made about these hedging strategies. First, many organized exchanges concurrently trade multiple contracts with different maturity dates. Thus, although the futures contract to be included into the portfolio, $\tau$, is exogenous to the above strategy, it should be the hedger's decision variable. The expressions (6) and (8) indicate that given the time of entry, $t$, and hedging horizon, $k$, the hedger should include into its portfolio the futures 
contract of which price change has the highest correlation with the spot price change. Because only a finite number of contracts are traded on any given day, one needs to calculate the optimal hedge ratio only for the futures contract with the highest correlation to the spot price.

Second, the time of entry, $t$, and hedging horizon, $k$, should be also endogenous to hedger's decision. The choice of $t$ and $k$ is important particularly for commodity with strong seasonality in mean price. Given a priori knowledge on such a seasonal pattern, hedgers should not hold a spot position from the peak to the off-peak demand season, for such practice would yield, at least on average, a negative return. (6) and (8) are optimal only given that the decision to carry over from $t$ to $t+k$ is predetermined. In practice, a position should be held only if the expected wealth, $\mathrm{E}\left[W_{t+k}\right]$, exceeds the value of risk associated with the minimum variance of portfolio return.

Finally, the expressions (6) through (9) indicate that both the optimal hedge ratio and the futures contract included into the portfolio depend on two attributes: (i) covariance of spot and futures price and (ii) variance of futures price changes. Apparently, specifications on the volatility dynamics of spot and futures prices play key roles in determining empirical estimates of these statistics and, hence, the optimal hedge strategy. We expect that the POTS model, allowing seasonal and cross-sectional variations in the factor loading and the variance of idiosyncratic error, will yield the optimal hedge ratio that varies by $\tau, t$, and $k$. In contrast, in conventional models of commodity price dynamics, factor loadings are determined by the time to maturity of contract and a small number of parameters defining the stochastic processes of underlying factors. Due to this restrictive specification, the optimal hedge ratio implied by these models does not vary by contract delivery date. In particular, a simple one-factor mean-reversion model considered by Schwartz (1997) and the two-factor model by Manoliu and Tompaids (2002) both imply that the optimal portfolio always includes the nearby contract. In other words, the specifications of stochastic processes of underlying factors stipulated in these models themselves determine the optimal hedging strategy. The regression models considered for the analysis of spot-futures price relationship are similarly incapable of implying cross-sectional and seasonal variations in the optimal hedge ratio due to a simple variance structure assumed for the disturbance term. 


\subsection{Optimal Hedging Strategy Implied by the POTS Model}

We evaluate the optimal hedge ratio based on the unconditional variance of daily price changes implied by the estimated POTS model. ${ }^{9}$ In doing so, we utilize the nearby futures price as a proxy for the spot price. ${ }^{10}$ We consider the case where $t$ and $k$ are predetermined and the hedger's decision is to choose the futures contract to be included into the portfolio, $\tau$, and the hedge ratio. ${ }^{11}$ We find the optimal solution for the two cases with different hedging horizons: (i) a header who carries a short position only for a single day $(k=1)$ and (ii) a header who carries a short position for one month, starting at the first day of each calendar month and ending at the last day of the same month.

From the estimated POTS model, the unconditional variance of daily futures price changes is given as,

$$
\mathrm{E}\left[\Delta \mathbf{F}_{t} \Delta \mathbf{F}_{t}^{\prime}\right]=\theta_{t} \theta_{t}^{\prime}+\lambda_{t} \lambda_{t}^{\prime}
$$

With the assumption $u_{d, t}$ iid $\sim \mathrm{N}(0,1)$, its diagonal and off-diagonal elements are,

$$
\begin{aligned}
& \mathrm{E}\left[\Delta F_{m, t}^{2}\right]=\theta^{n}(m-t)^{2}+\lambda^{m}(m-t)^{2} \\
& \mathrm{E}\left[\Delta F_{m, t} \Delta F_{\mu, t}\right]=\theta^{n}(m-t) \theta^{\mu}(\mu-t) \quad \text { for } \mu, m>t \text { and } m \neq \mu .
\end{aligned}
$$

Using the expressions (11), the correlation between two futures prices for delivery $\tau_{1}$ and $\tau_{2}$ is,

$$
\rho_{t, \tau_{1}, \tau_{2}}=\frac{\theta^{\tau_{1}}\left(\tau_{1}-t\right) \theta^{\tau_{2}}\left(\tau_{2}-t\right)}{\left(\theta^{\tau_{1}}\left(\tau_{1}-t\right)^{2}+\lambda^{\tau_{1}}\left(\tau_{1}-t\right)^{2}\right)^{\frac{1}{2}}\left(\theta^{\tau_{2}}\left(\tau_{2}-t\right)^{2}+\lambda^{\tau_{2}}\left(\tau_{2}-t\right)^{2}\right)^{\frac{1}{2}}}=\pi_{\tau_{1}} \pi_{\tau_{2}}
$$

\footnotetext{
${ }^{9}$ One can also evaluate the optimal hedge ratio using the model's implied conditional variance. Unlike the unconditional variance used in the main text, the conditional variance depends on the historical movements of the natural gas futures price. We only present evaluations based on the unconditional variance, because our objective here is to draw a general implication about the need to model seasonality.

${ }^{10}$ To examine the optimal hedging strategy involving the hedger's position in spot market requires an appropriate picture on the volatility dynamics of the spot price. While one can modify the POTS model to include the daily spot price changes into its system, the martingale assumption does not allow deterministic variations in spot price. One can relax this assumption, yet, the seasonal mean price is difficult to estimate because the dynamics of spot price does not have a closed-form solution due to non-negativity of storage.

${ }^{11}$ The optimal strategy involving choices of the time of entry, $t$, and hedging horizon, $k$, should require the expected return to such a portfolio.
} 
where $\pi_{\tau_{i}}=\theta^{\tau_{i}}\left(\tau_{i}-t\right)\left(\theta^{\tau_{i}}\left(\tau_{i}-t\right)^{2}+\lambda^{\tau_{i}}\left(\tau_{i}-t\right)^{2}\right)^{-\frac{1}{2}}$ is the square root of the share of the total variance of contract $i$ explained by the common factor. (12) indicates that the variance of the portfolio return is minimized when the portfolio includes the futures contract for which the largest share of price change is accounted for by the common factor.

The optimal hedge ratio and the minimized variance are,

$$
\begin{gathered}
\eta_{t, \tau_{1}, \tau_{1}}^{*}=\frac{\theta^{\tau_{1}}\left(\tau_{1}-t\right) \theta^{\tau_{2}}\left(\tau_{2}-t\right)}{\theta^{\tau_{2}}\left(\tau_{2}-t\right)^{2}+\lambda^{\tau_{2}}\left(\tau_{2}-t\right)^{2}} \\
\mathrm{~V}\left[W_{t+k}\right]=\left(\theta^{\tau_{1}}\left(\tau_{1}-t\right)^{2}+\lambda^{\tau_{1}}\left(\tau_{1}-t\right)^{2}\right)\left(1-\rho_{t, \tau_{1}, \tau_{2}}^{2}\right) Q^{2}
\end{gathered}
$$

For the hedging strategy with horizon over $t_{1}$ to $t_{2}$, we need the expressions for the unconditional variance of daily futures price changes, which, with the martingale property assumed in the model, is simply the sum of the daily price changes over this period,

$$
\mathrm{E}\left[\Delta \mathbf{F}_{t_{1}, t_{2}} \Delta \mathbf{F}_{t_{1}, t_{2}}{ }^{\prime}\right]=\sum_{s=t_{1}+1}^{t_{2}}\left(\boldsymbol{\theta}_{s} \boldsymbol{\theta}_{s}{ }^{\prime}+\boldsymbol{\lambda}_{s} \boldsymbol{\lambda}_{s}{ }^{\prime}\right)
$$

with its elements,

$$
\begin{aligned}
& \mathrm{E}\left[\Delta F_{m, t_{1}, t_{2}}^{2}\right]=\sum_{s=t_{1}+1}^{t_{2}} \theta^{m}(m-s)^{2}+\lambda^{m}(m-s)^{2} \\
& \mathrm{E}\left[\Delta F_{m, t_{1}, t_{2}} \Delta F_{\mu, t_{1}, t_{2}}\right]=\sum_{s=t_{1}+1}^{t_{2}} \theta^{m}(m-s) \theta^{\mu}(\mu-s) \quad \text { for } m, \mu>t_{2}, m \neq \mu .
\end{aligned}
$$

The correlation between the two contracts over the horizon $t_{1}$ to $t_{2}$ is,

$$
\rho_{t_{1}, t_{2}, \tau_{1}, \tau_{2}}=\frac{\sum_{s=t_{1}+1}^{t_{2}} \theta^{\tau_{1}}\left(\tau_{1}-s\right) \theta^{\tau_{2}}\left(\tau_{2}-s\right)}{\left(\sum_{s=t_{1}+1}^{t_{2}} \theta^{\tau_{1}}\left(\tau_{1}-s\right)^{2}+\lambda^{\tau_{1}}\left(\tau_{1}-s\right)^{2}\right)^{\frac{1}{2}}\left(\sum_{s=t_{1}+1}^{t_{2}} \theta^{\tau_{2}}\left(\tau_{2}-s\right)^{2}+\lambda^{\tau_{2}}\left(\tau_{2}-s\right)^{2}\right)^{\frac{1}{2}}}
$$

The optimal hedge ratio and the associated minimum variance are, 


$$
\begin{gathered}
\eta_{t_{1}, t_{2}, \tau_{1}, \tau_{1}}^{*}=\frac{\sum_{s=t_{1}+1}^{t_{2}} \theta^{\tau_{1}}\left(\tau_{1}-s\right) \theta^{\tau_{2}}\left(\tau_{2}-s\right)}{\sum_{s=t_{1}+1}^{t_{2}} \theta^{\tau_{2}}\left(\tau_{2}-s\right)^{2}+\lambda^{\tau_{2}}\left(\tau_{2}-s\right)^{2}} \\
\mathrm{~V}\left[W_{t_{1}, t_{2}, \tau_{1}, \tau_{1}} \mid \eta_{t_{1}, t_{2}, \tau_{1}, \tau_{1}}^{*}\right]=\sum_{s=t_{1}+1}^{t_{2}}\left(\theta^{\tau_{1}}\left(\tau_{1}-s\right)^{2}+\lambda^{\tau_{1}}\left(\tau_{1}-s\right)^{2}\right)\left(1-\rho_{t_{1}, t_{2}, \tau_{1}, \tau_{2}}^{2}\right) Q^{2}
\end{gathered}
$$

Equations (13) and (18) indicate that the optimal hedge ratio is the function of time to entry, $t$, hedging horizon, $k$, and delivery period of the futures contract in the portfolio, $\tau_{2}$. It particular, the optimal hedge ratio increases with the variance of the nearby futures price that is attributable to the common factor and/or the proportion of the futures price variance explained by the common factor. In other words, a hedger should take a large short position when the nearby futures price is very volatile and it is related to the futures prices of subsequent delivery. Even when the nearby futures price is very volatile, a hedger's position is small if its price movement is not closely related to the other concurrently traded contracts.

\subsection{Results}

Figures 5 through 7 illustrate the futures contract included into the optimal portfolio, the optimal hedging ratio, and the minimum variance attained by the optimal portfolio for each of the two hedging horizons. First, for a daily hedging strategy, the optimal portfolio frequently includes four contracts: the December contracts for the period between mid May and mid August and either the June, July, or August contract for the period between mid September to mid April in the following year. These contracts are often used because they exhibit the highest share of their price volatility explained by the common factor in relevant period. Other contracts are rarely included into the portfolio. Because of the Samuelson effects, the optimal portfolio never includes the short-dated contracts. The three-month-ahead contract is the shortest horizon and is used only in the first half of April and the first half of August where the variance minimizing contract switches gradually from the August to winter and from the December to summer contracts, respectively.

The predominance of the June, July, August, and December contracts is consistent with our previous discussion relating seasonality of price volatility to that of natural gas demand and storage. From the beginning of the year, the optimal portfolio includes the July contract and then switch to the August contract. Contracts for earlier maturity are not used due to their high 
idiosyncratic volatility. This high idiosyncratic volatility represents the Samuelson effect for the March contract whereas it is due to low gas storage for the April to June contracts - price movements of these contracts in response to demand, supply, and other market shocks are not linked to one another through available storage.

In the middle of April, the optimal contract for hedging purposes switches from the August to distant contracts as the August contract becomes subject to the maturity effect. This transition is rather quick with the August contract replaced by the December contracts by mid May. The September through November contracts are used only for a short duration because of their high idiosyncratic variance. The next transition takes place in the middle of August when the optimal portfolio switches to the March contract as idiosyncratic variance of the December contract increases rapidly. The January and February contract are not used in this transition, because a large share of their price variation is contract-specific. The March and April contract are optimal only for a short period because price movements of these two contracts are contract-specific due to the low inventory applicable during those two months.

Figure 6a plots the optimal hedge ratio as a function of trading date. As shown in (13), the optimal hedge ratio is simply the ratio of the covariance between the nearby and the futures contract to the variance of the futures contract included in the portfolio. In the figure, the optimal hedge ratio is always above one, indicating that the covariance always exceeds the variance of the futures contract. This is because price of the futures contract included into the optimal portfolio is at least three months away from delivery and, hence, displays little variation, a dominant share of which reflects the common factor. (Whether a hedge ratio above makes sense is a question for the whole theory of "optimal hedging.") The optimal hedge ratio is particularly high in January and December, during which the price variance of the nearby contract is largely attributable to the common factor, yielding large covariance. Even though the nearby futures contract exhibits high price volatility from September to November, the optimal hedge ratio is only moderate in these months because much of the price variation is contract specific and, hence, has low covariance.

Figure $\mathbf{6 b}$ plots the optimal hedge ratio for the portfolio including the second position contract, which is the optimal solution according to the one- and two-factor models considered 
by Manoliu and Tompades (2002). ${ }^{12}$ Two observations become clear with this comparison of the two hedging strategies. First, the optimal hedge ratio is substantially lower for the portfolio including the second position than for the optimal portfolio implied by the POTS model. This is simply because the share of the price variance explained by the common factor is smaller for the second position than for more distant contract. Second, the optimal hedge ratio decreases as the contract approaches to its maturity. This is, again, because the share of the price variance explained by the common factor decreases due to the Samuelson effect. These results are peculiar to the mean-reversion process assumed for the underlying factors in models of Manoliu and Tompades (2002). This particular stochastic process assures that factor loading increases monotonically as the contract approaches its maturity date. Thus, the price correlation is always the highest for the two contracts with the minimum distance in their maturity dates. In other words, the specification of stochastic processes of the underlying factors itself determines the futures contract to be included in the optimal portfolio.

Figure $7 \mathbf{a}$ illustrates the variance of the optimal portfolio in ratio to the variance of the unhedged portfolio, i.e., the total variance of the nearby futures price. The figure shows that the optimal hedging strategy implied by the estimated POTS model reduces price risk substantially. The variance is reduced to less than $40 \%$ of the variance of the nearby futures, except that it is above $40 \%$ in October, November and February. The magnitude of variance reduction is small in these three months as the nearby futures prices (the November, December, and March contracts) are inherently very volatile and their price volatility is contract specific. Quite noticeably, the variance of the optimal portfolio is below $35 \%$ of the variance of the nearby futures price for December and January during which the nearby futures (January and February contract) prices are very volatile. For each of the 12 months, the minimum variance attained by the optimal portfolio is higher toward the end of each month, simply because the Samuelson effect raises the contract specific volatility of the nearby futures.

Figure $7 \mathbf{b}$ compares the optimal hedging strategy implied by the POTS model with the straightforward strategy of utilizing the second-position contract. The figure indicates that the strategy based on the POTS model attains the portfolio variance that is about 15 to 45 percent below the variance of the portfolio utilizing the second position contract. This is because the

\footnotetext{
${ }^{12}$ See Tables 4 and 5 on pages 38 and 39 of Manoliu and Tompaids (2002). Numbers in Figure 6b were calculated by using the volatility estimates of the POTS model in (13).
} 
futures contract included in the former strategy is less subject to contract specific variations than in the second-position contract.

The optimal hedging strategies for a monthly horizon yield essentially the same results as those for a daily horizon. Three summer contracts (June, July and August) and the December contract are the most commonly included into the optimal portfolio whereas the rest of the futures contracts are less common with the October and November contract used in April and May, respectively, and the January and April contracts in August and September. The optimal hedging ratio and the minimum variance attained by the optimal contact are also similar to those for daily hedging strategy. They are almost identical to the monthly averages of their corresponding values for daily hedging horizon - price risk is reduced into half the size of price variance of the nearby contract by high hedge ratio, ranging between 1.2 and slightly above 2.0, which is due to a small variance of the futures contract included into the portfolio.

\section{CONCLUSION}

We have examined the volatility dynamics of the NYMEX natural gas futures prices by the partially overlapping time-series (POTS) model of Smith (2005). The estimated POTS model reveals that the NYMEX natural gas futures prices exhibit time-to-maturity effects and strong seasonal variations in their price volatility - volatility rapidly increases in the last three months of trading period and is higher for winter contracts than for spring and summer contracts. In addition, our analysis has revealed that the persistence of price shocks and, hence, the correlation of concurrently traded contracts in their daily price changes exhibit substantial seasonal and cross-sectional variations. Specifically, price volatility is relatively high in two trading periods: early November to mid January for the January to April contracts and early May to September for all contracts maturing before the following March. Such volatility dynamics are closely related to the seasonal pattern of the US natural gas storage in a way consistent with the theory of storage.

The depicted portrait of natural gas price volatility dynamics implies that a trader in need of hedging their spot (nearby futures) price risk should cross hedge with a futures contract of at least three months to maturity to avoid high contract-specific volatility representing the Samuelson effect. In addition, they should include into their portfolio the December contract to hedge against spot price risk during spring and summer months and either of the June, July, and 
August contract in winter months. The optimal hedge ratio is high, ranging from 1.2 to slightly above 2.0, because the price of the futures contract exhibits much smaller movement than the nearby contract while they share much information regarding underlying market conditions.

These results suggest that the previous studies of the spot-futures price relationships and the dynamics of natural gas futures prices are subject to misspecification bias in the variance structure of the disturbance terms in their regression models. In particular, models of commodity dynamics should allow more flexible specifications, in particular, seasonal and cross-sectional variations in the factor loadings and the variance of the idiosyncratic error. Also, the analysis of spot-futures price relationships should allow seasonal and cross-sectional variation in the variance of the disturbance term. The assumption of constant, more specifically, zero, basis is clearly inappropriate, for the cost of carry is not constant for storable commodity with strong seasonality in demand and/or supply. The optimal hedging strategies implied by these misspecified models are noticeably ineffective with the variance of the portfolio return 18 to $80 \%$ higher than the minimum variance attained by the hedging strategy suggested by the estimated POTS model.

\section{REFERENCES}

Energy Information Administration. 2002. Derivatives and Risk Management in the Petroleum, Natural Gas, and Electricity Industries. Energy Information Administration, U.S. Department of Energy, Washington, DC.

Engle, R. F., and Kroner, K. F. 1985. "Multivariate simultaneous generalized ARCH," Econometric Theory, 11:122-150.

Hamilton, J. D. 1994. "State-Space Models," in Engle, R. F., and McFadden, D. L. eds. Handbook of Econometrics, Vol. 4. Elsevier, Amsterdam.

Lien, D., and Root, T. H. 1999. "Convergence to the long-run equilibrium: the case of natural gas markets," Energy Economics, 21: 95-110.

Lucia, J. J., and Schwartz, E. S. 2002. "Electricity prices and power derivatives: Evidence from the Nordic Power Exchange," Review of Derivatives Research, 5: 5-50.

Manoliu, M., and Tompaidis, S. 2002. "Energy futures prices: Term structure models with Kalman filter estimation," Applied Mathematical Finance, 9: 21-43.

Movassagh, N., and Modjtahedi, B. 2005. "Bias in backwardation in natural gas futures prices," Journal of Futures Markets, 25: 281-308. 
Modjtahedi, B. and Movassagh, N. 2005. "Natural gas-futures: Bias, predictive performances, and the theory of storage," Energy Economics, 27: 617-637.

Root, T. H., and Lien, D. 2003. "Can modeling the natural gas futures market as a threshold cointegrated system improve hedging and forecasting performance?" International Review of Financial Analysis, 12: 117-131.

Schwartz, E. S. 1997. “The stochastic behavior of commodity prices: Implications for valuation and hedging," Journal of Finance, 52: 923-973.

Smith, A. 2005. "Partially overlapping time-series model: A new model for volatility dynamics in commodity futures," Journal of Applied Econometrics, 20:405-422.

Suenaga, H. 2005. "Analysis of spot-forward price relationships in the restructured electricity markets," Ph.D. Dissertation, University of California, Davis.

Suenaga, H., and Williams, J. C. 2005. "Simulation-based analysis of the efficiency of the NYMEX natural gas market," Proceedings to the 25th USAEE/IAEE North American Conference.

Walls, W. D. 1995. "An econometric analysis of the market for natural gas futures," Energy Lournal, 16: 71-83.

Williams, J. C., and Wright, B. D. 1991. Storage and Commodity Markets. Cambridge University Press, New York. 
Table 1. Maximum Likelihood Estimates of GARCH Parameters

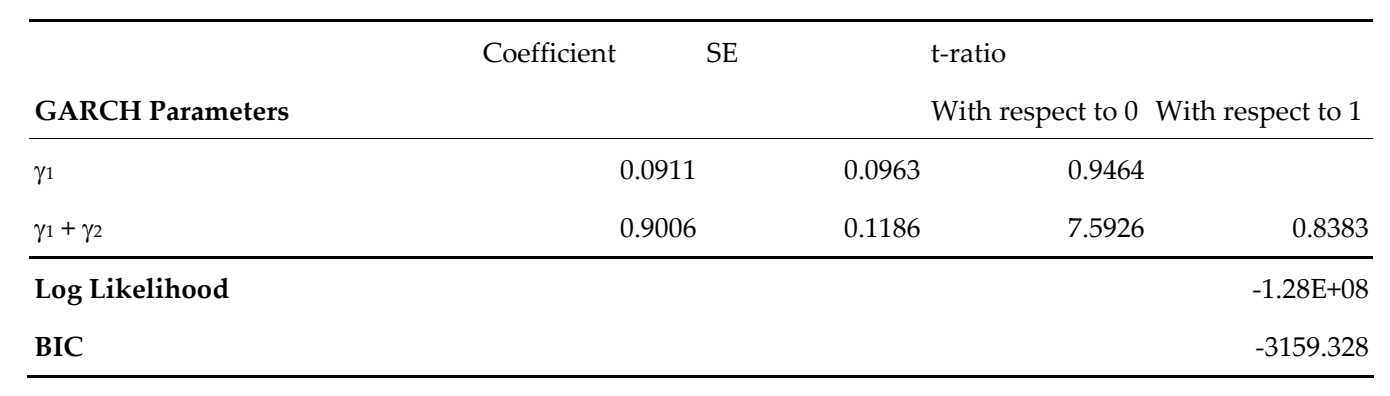

Table 2. Proportion of the Variance Explained by a Common Factor

\begin{tabular}{lcc}
\hline Overall & & 0.8257 \\
\hline By contract & & \\
& 1 & 0.7818 \\
& 2 & 0.8135 \\
& 3 & 0.7821 \\
& 4 & 0.8580 \\
& 5 & 0.8643 \\
& 6 & 0.8605 \\
7 & 0.8617 \\
& 8 & 0.8834 \\
9 & 0.8620 \\
10 & 0.8411 \\
11 & 0.8088 \\
12 & 0.7347 \\
\hline
\end{tabular}


Figure 1. Close price of NYMEX natural gas futures price as of April 1, 2003

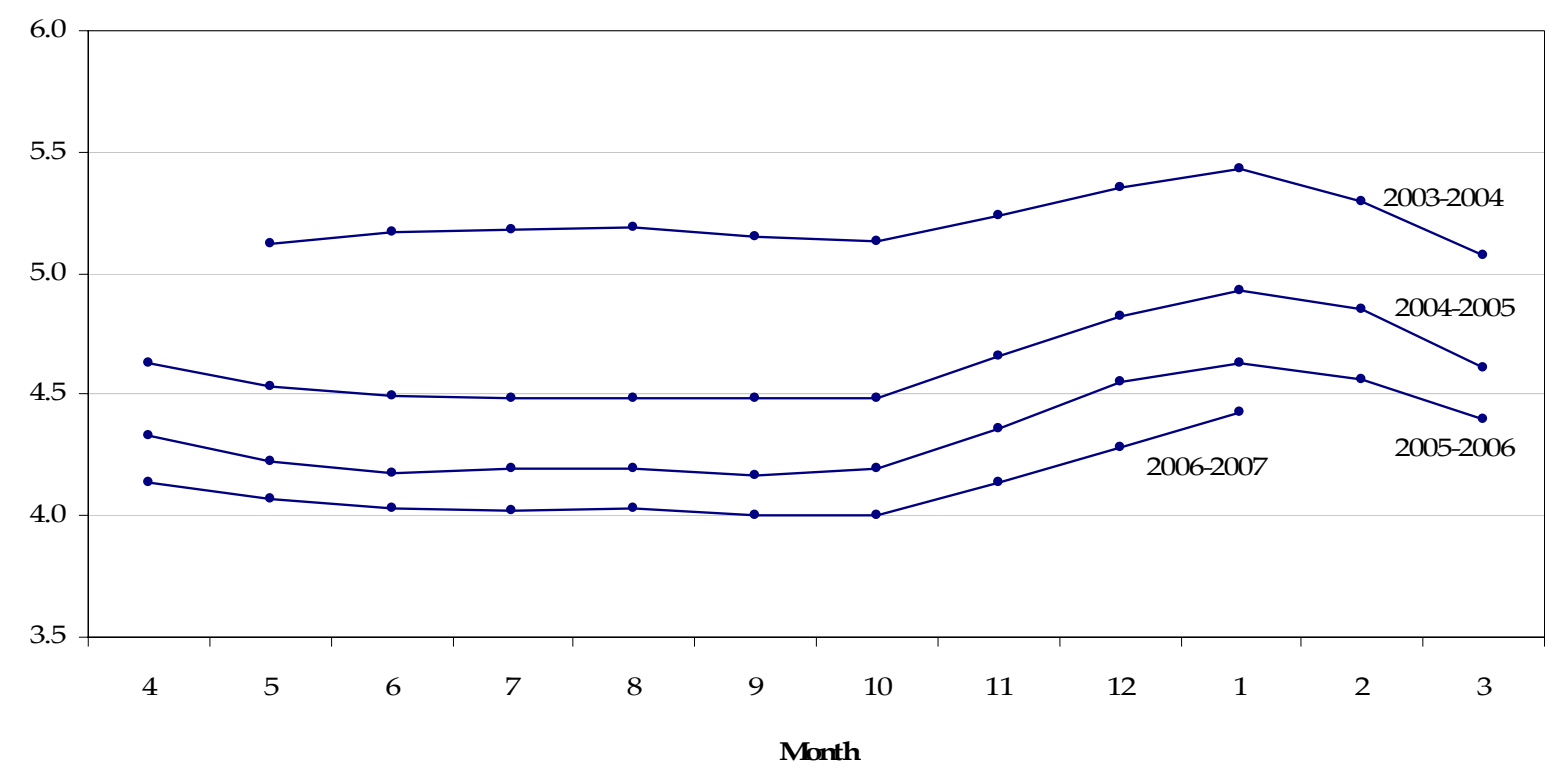


Figure 2. Variance of Daily Log Price Chnages Implied by the Estimated POTS Model

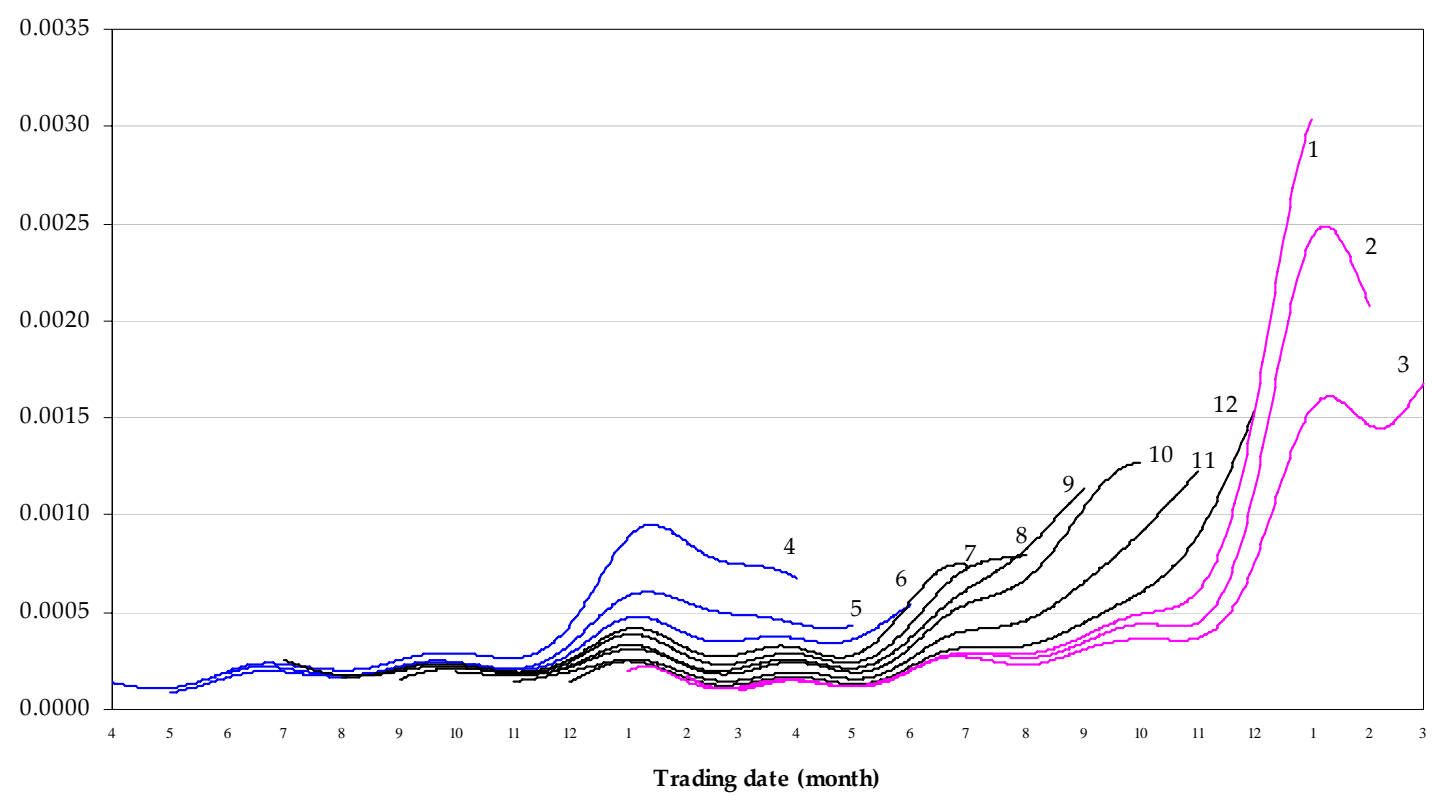

Figure 3. Share of Price Variation Explained by the Common Factor

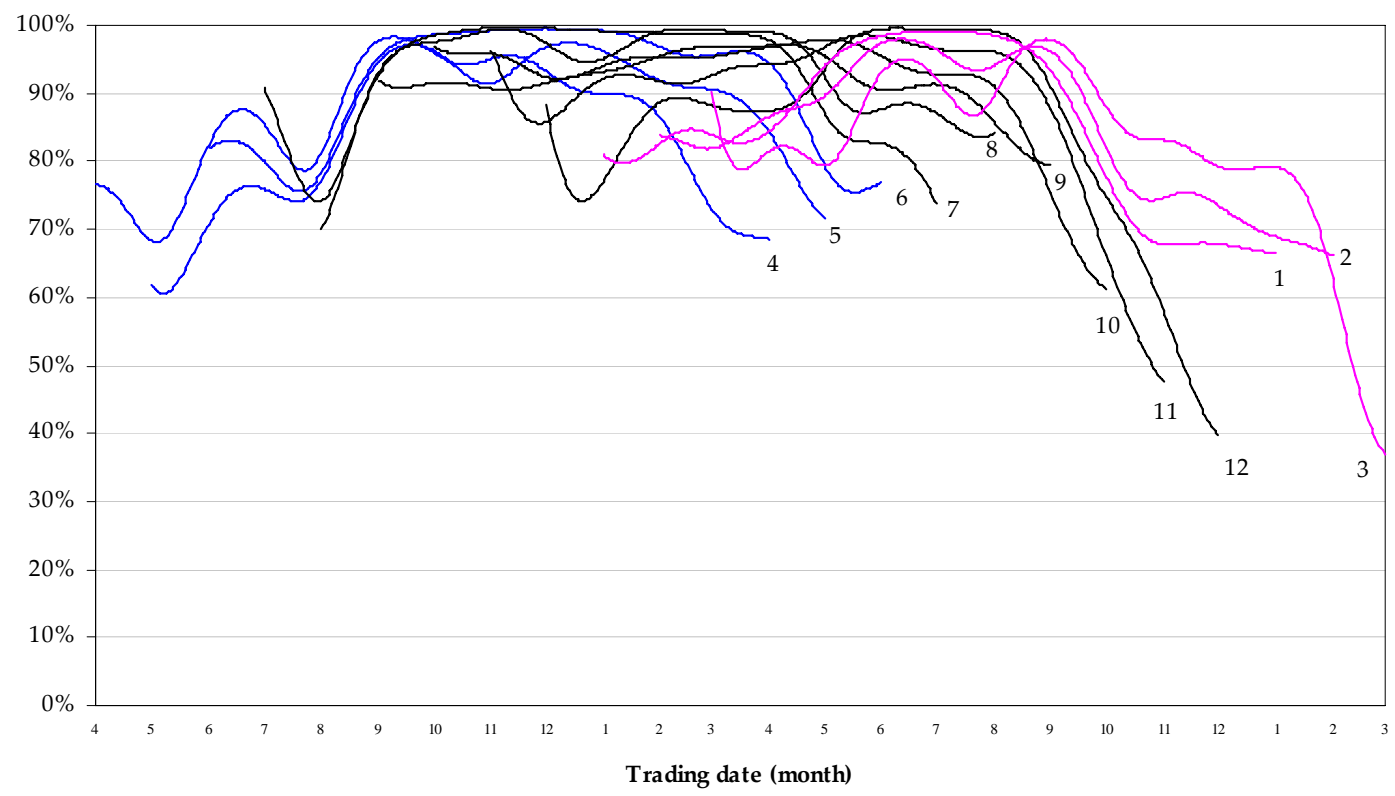


Figure 4. US Natural Gas Underground Storage - Monthly Average of Working Gas for 1976 - 2005

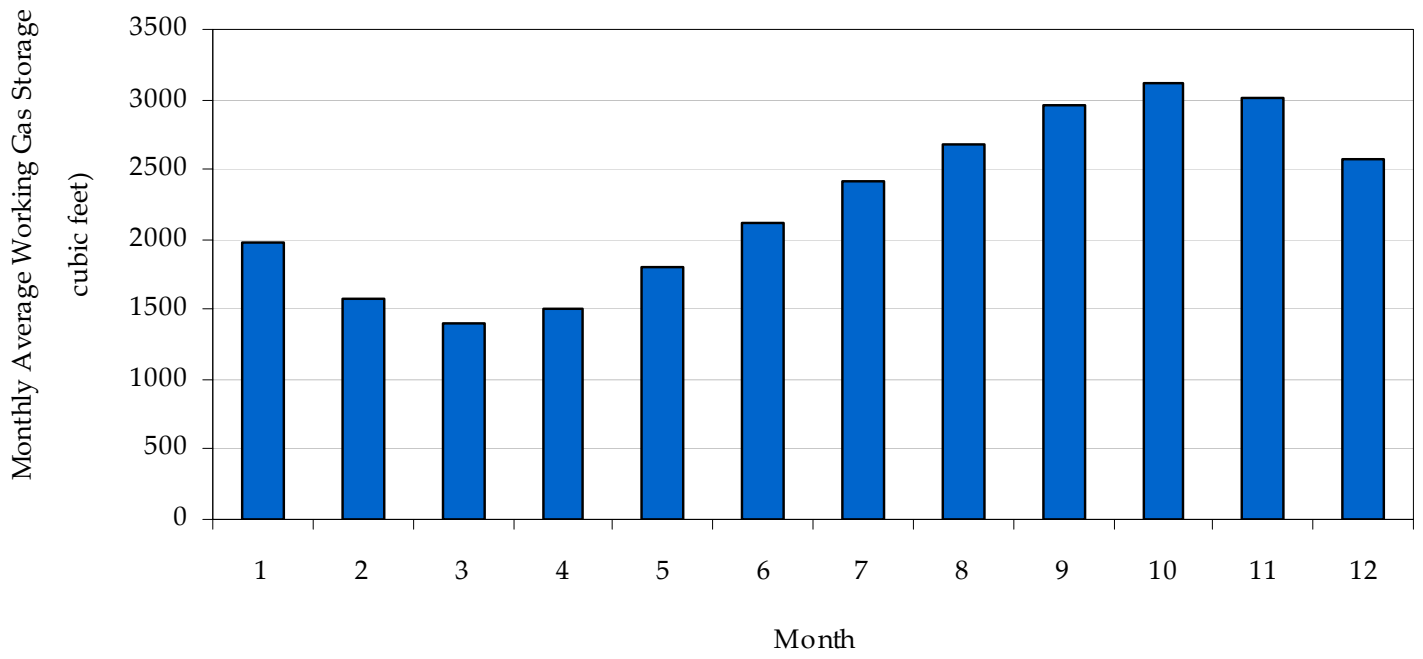


Figure 5. Delivery Month of the Futures Contract Included in the Optimal Portfolio

$\square$ Monthly — Daily

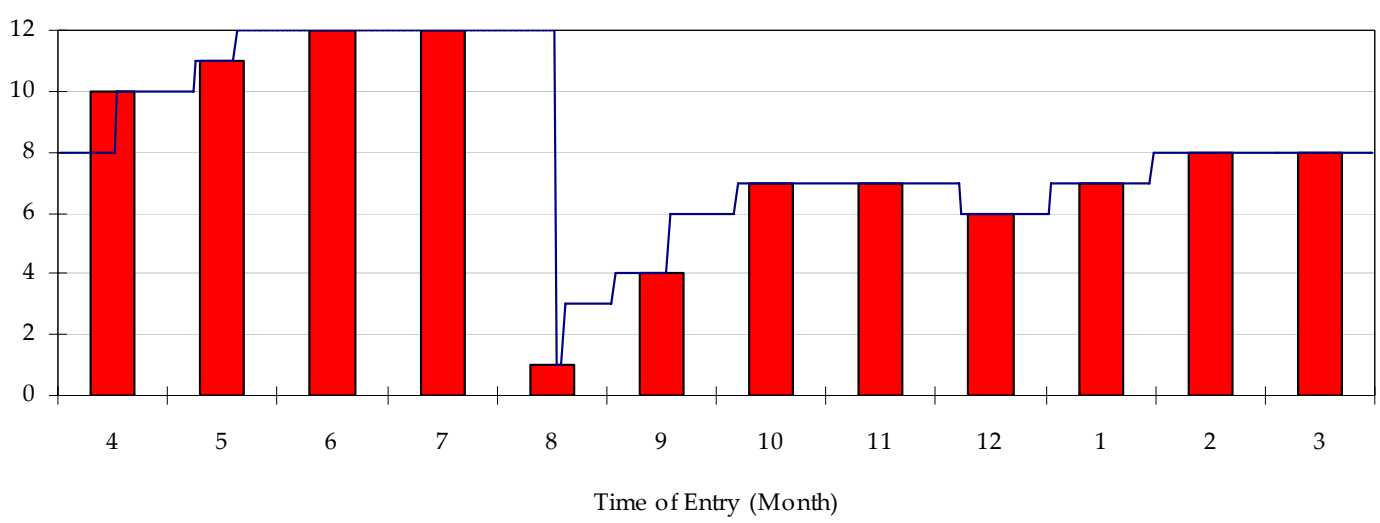

Figure 6a. Optimal Hedge Ratio

$\square$ Monthly —Daily

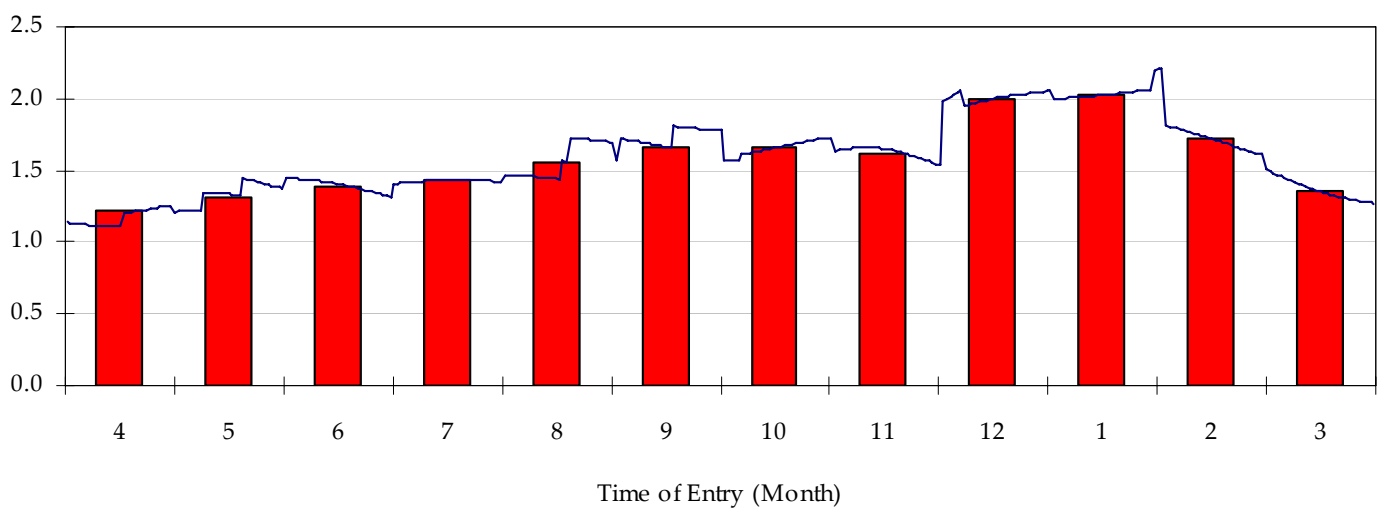

Figure 6b. Optimal Hedge Ratio for Portfolio w/ Nearby Contract

Monthly —Daily

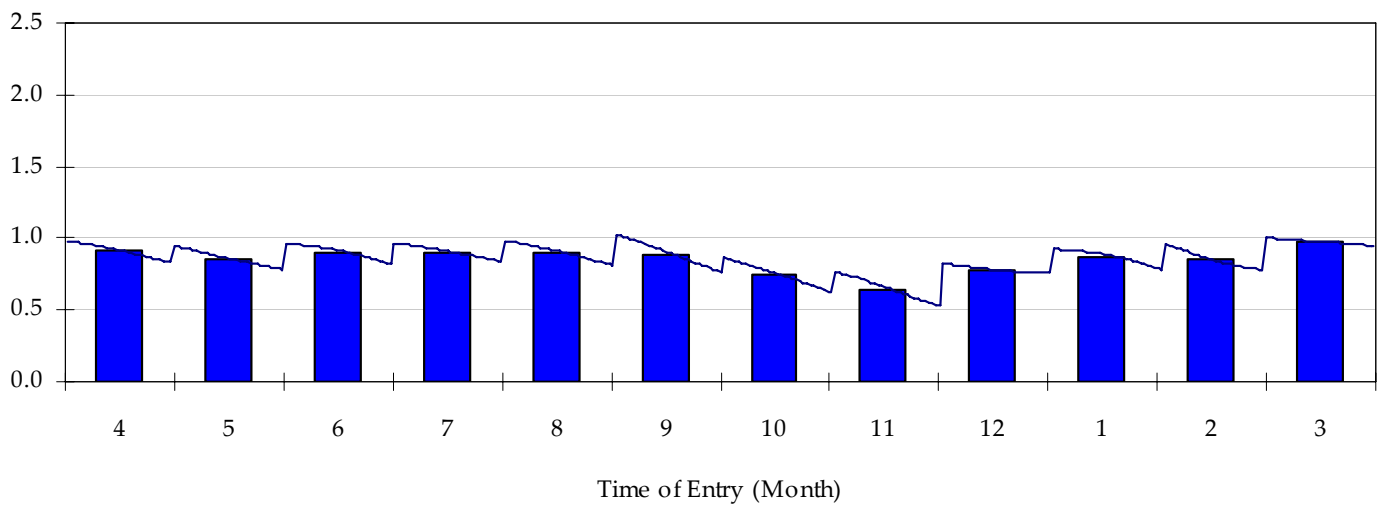


Figure 7a. Minimum Variance Relative to the Variance of Unhedged Portfolio

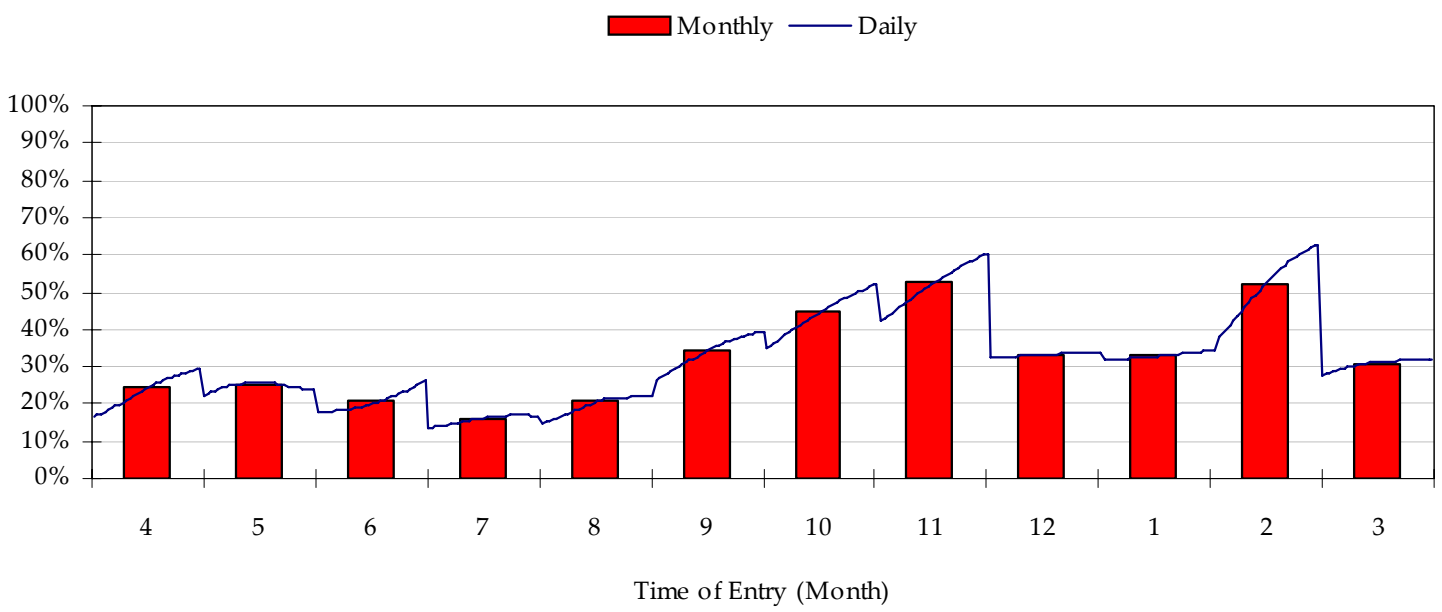

Figure $7 \mathrm{~b}$. Minimum Variance Relative to the Variance of Portfolio with Second Position

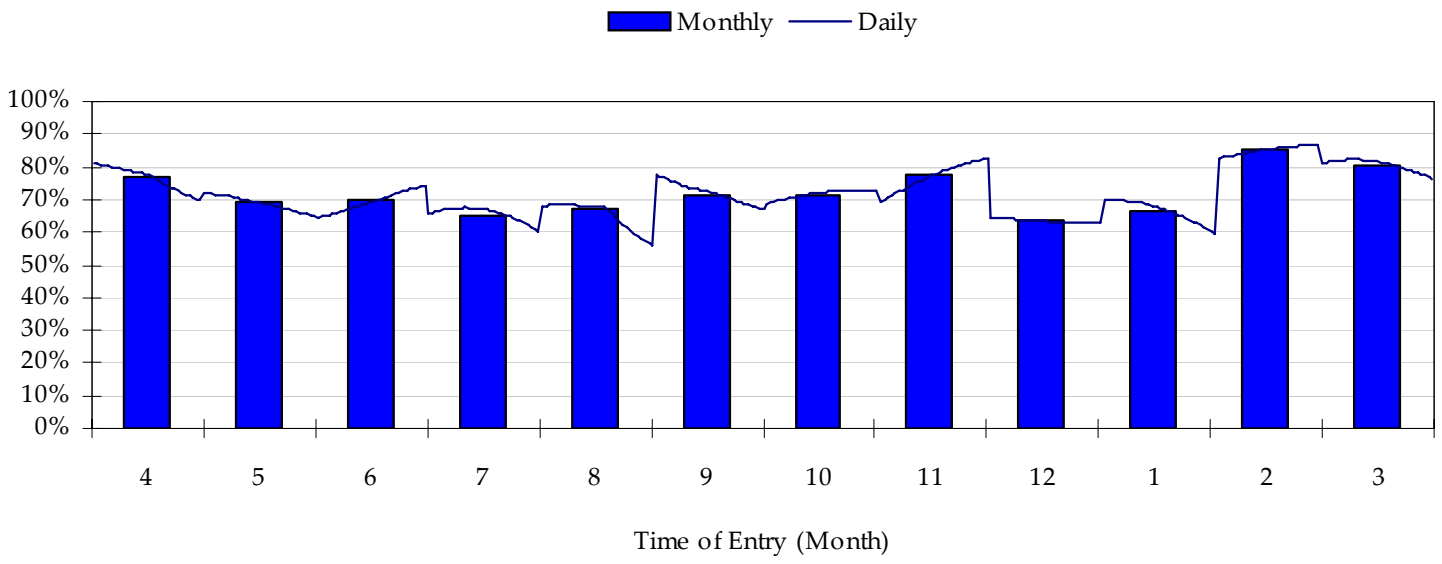

